\title{
Efficacy and safety of levodropropizine and dihydrocodeine on nonproductive cough in primary and metastatic lung cancer
}

\author{
G. Luporini*, S. Barni**, E. Marchi+, L. Daffonchio++
}

Efficacy and safety of levodropropizine and dihydrocodeine on nonproductive cough in primary and metastatic lung cancer. G. Luporini, S. Barni, E. Marchi, L. Daffonchio. CERS Journals Ltd 1998.

ABSTRACT: Nonproductive cough is a frequent and distressing symptom in patients with lung cancer, and it is not even relieved by palliative chemotherapy.

A double-blind, randomized clinical trial regarding the treatment of nonproductive cough was performed in 140 adults with primary lung cancer or metastatic cancer of the lungs. The therapeutic efficacy and the tolerability of a 7-day treatment with levodropropizine drops $(75 \mathrm{mg}$ t.i.d.) were evaluated in comparison with dihydrocodeine drops (10 $\mathrm{mg}$ t.i.d.; 7 days). Efficacy was assessed on the basis of cough severity scores, number of night awakenings due to cough, and overall estimate of antitussive efficacy. Tolerability was evaluated by laboratory results, vital signs and any adverse event occurring during the clinical trial, including presence or absence of somnolence.

Subjective cough severity was significantly reduced during treatment with either levodropropizine and dihydrocodeine, the antitussive effect and its time-profile being similar for both drugs. Also, according to the investigator's evaluation, both levodropropizine and dihydrocodeine produced a significant decrease in cough severity. Concurrently with the relief of cough, the number of night awakenings was decreased significantly by both drugs, with no difference between the two treatments. No change in laboratory test values was considered clinically relevant, and vital signs were not clinically affected. The number of patients reporting adverse events was similar in the levodropropizine $(n=6)$ and dihydrocodeine $(n=4)$ group. However, the percentage of patients experiencing somnolence in the group receiving levodropropizine $(\mathbf{8 \%})$ was significantly lower as compared with that of the dihydrocodeine group $(22 \%)$.

These results confirm the antitussive effectiveness of levodropropizine and suggest a more favourable benefit/risk profile when compared to dihydrocodeine. Eur Respir J 1998; 12: 97-101.
*Divisione di Oncologia Medica, Ospedale San Carlo Borromeo, Milan, Italy **Day Hospital - Oncologia Medica, Ospedale S. Gerardo, Monza (MI), Italy ${ }^{+}$Centro per lo Studio delle Broncopneumopatie Senili, Casatenovo (LC), Italy. ${ }^{++}$Research \& Development Dept, Dompé s.p.a, Milan, Italy.

Correspondence: G. Luporini

Divisione di Oncologia Medica

Ospedale San Carlo Borromeo

Via Pio II, 3

20153 Milan

Italy

Fax: 39240222323

Keywords: Antitussives

cough

dihydrocodeine

levodropropizine

lung cancer

somnolence

Received: December 41997

Accepted after revision February 151998

The trial was sponsored by Dompé farmaceutici s.p.a.
Cough is a frequent symptom in patients with lung cancer $[1,2]$, regardless of the histological type $[1,3]$. Cough is more distressing in patients who continue to smoke and tends to worsen inexorably with time [4]. Chemotherapy can reduce cough in approximately two-thirds of patients [5], but the effect is short and does not persist after therapy discontinuation [6]. Moreover, cough is not even relieved by palliative treatment in most patients [2, 4]. Therefore, to improve the quality of life of patients with neoplastic cough, it is often necessary to administer antitussive agents.

Opioid drugs such as codeine derivatives, although frequently used in the management of cough in these patients, have relevant effects on cognitive and psychomotor function $[6,7]$. These antitussive drugs act by directly modulating the central mechanisms of cough, an effect possibly mediated through an increase in 5-hydroxy-tryptamine (5-HT) release in the brain stem [8]. Although opioids are still widely used in the management of cough in patients with lung cancer, it is certain that drugs acting on peripheral mechanisms of cough and with poor or absent central activity should be preferable in the treatment of neoplastic cough.

Levodropropizine, a phenylpiperazinopropane derivative, is the (-) enantiomer of dropropizine. This nonopioid compound is effective against cough induced by different agents in animal models [9, 10]. Several experimental evidences indicate that levodropropizine acts through a peripheral mechanism, mainly involving modulation of sensitive C-fibre activity [11]. This mechanism of action might account for the pharmacological profile observed in clinical practice. Indeed, levodropropizine proved to be an effective drug against cough associated with different lung diseases in both children and adults [11-13], while being generally devoid of central side-effects.

In this study, we examined the efficacy and safety of a short-term treatment with levodropropizine compared to dihydrocodeine rhodanate in patients affected by persistent nonproductive cough due to primary lung cancer or metastatic cancer to the lungs. 


\section{Methods}

\section{Population}

We selected in- and outpatients of either sex older than $18 \mathrm{yrs}$, with a diagnosis of unilateral or bilateral, primary lung cancer or metastatic cancer to the lungs. Patients with a documented history of lung cancer who developed persistent, nonproductive cough were enrolled if they demonstrated at least 5 coughs $\cdot \mathrm{h}^{-1}$.

Exclusion criteria were: presence of productive cough; presence of other diseases causing cough such as bronchial asthma, bronchiectasis, tuberculosis, heart failure; and treatment with other antitussives, angiotensin-converting enzyme (ACE) inhibitors, antihistamines, systemic sedatives, or mucoactive drugs.

The study was conducted in agreement with the European Economic Community (EEC) Guideline on Good Clinical Practice (GCP), and the protocol was approved by an independent Regional Ethics Committee and by the Local Ethics Authority of each hospital. Before enrolment, signed or witnessed informed consent to participate into the study was obtained by each patient.

\section{Study design}

We conducted a multicentre, parallel group, doubleblind study. After the preliminary evaluation, patients were randomized to treatment with levodropropizine (Levotuss ${ }^{\circledR}$, Dompé Farmaceutica, Milan, Italy, 6\% oral drops) or dihydrocodeine rhodanate (Paracodina ${ }^{\circledR}$, Knoll Farmaceutica, Milan, Italy, $1 \%$ oral drops). Daily administered doses were equal to $75 \mathrm{mg}$ ( 25 drops) t.i.d. of levodropropizine and $10 \mathrm{mg}$ ( 25 drops) t.i.d. of dihydrocodeine. Each dose was scheduled at $6 \mathrm{~h}$ to $8 \mathrm{~h}$ intervals (between 07:00 and 09:00 $\mathrm{h}$, at 15:00 $\mathrm{h}$ and between 21:00 and 23:00 h). The dose of dihydrocodeine (10 $\mathrm{mg}$ t.i.d.) was set on the basis of the therapeutic recommended daily dosage. To keep the two treatments indistinguishable as per the number of drops dispensed, the dose of levodropropizine $(75 \mathrm{mg}$ t.i.d.) was slightly higher than the recommended dose (60 $\mathrm{mg}$ t.i.d.). Treatments were administered for 7 days, a period that allowed sufficiently accurate estimate of drug efficacy in patients with such severe disease.

\section{Efficacy}

Efficacy was assessed on the basis of cough severity graded by both the patients and the investigators according to the following five-point scale: 1 for absent $(0-1$ cough $\left.\cdot \mathrm{h}^{-1}\right) ; 2$ for mild $\left(2-4\right.$ coughs $\left.\cdot \mathrm{h}^{-1}\right) ; 3$ for moderate $(5-$ 10 coughs $\left.\cdot \mathrm{h}^{-1}\right) ; 4$ for severe $\left(11-20\right.$ coughs $\left.\cdot \mathrm{h}^{-1}\right)$; and 5 for very severe $\left(>20\right.$ coughs $\left.\cdot h^{-1}\right)$ cough. In particular, the number of coughs was recorded by the patient for five consecutive hours, either immediately after the first or second daily dose, and by the investigator after observing the patients for $15 \mathrm{~min}$. The cough severity was evaluated at baseline, and on days 1, 3 and 7 during treatment. Patients also recorded the number of night awakenings due to cough before treatment and on days 1, 3 and 7 during treatment.
At the end of the 7-day treatment, about two-thirds of the patients and their investigators were asked to give an overall estimate of the antitussive efficacy of the treatment according to the following four-point scale: worsening of cough; no change in cough; improvement of cough; and disappearance of cough.

\section{Safety}

The primary variable for safety was the presence of somnolence, defined as a decrease of the waking status during the day and monitored by specific questioning (no daytime somnolence; mild daytime somnolence; and severe daytime somnolence).

The secondary variables for safety were data on haematology, blood chemistry, urinalysis, blood pressure and heart rate, recorded before entry and at the end of treatment. Any adverse event was also recorded throughout the study period.

\section{Statistical analysis}

The appropriate descriptive statistics were performed on all collected variables to describe the sample. To ensure homogeneity between the two treatment groups, a $\chi^{2}$ test or Student's t-test was applied.

Cough severity and the number of awakenings at night were analysed by ANOVA (split-plot model). For the latter parameter, only data from patients with at least one wakeup at baseline were considered. The presence of somnolence was analysed by the $\chi^{2}$ test. Missing data were processed as such, since no replacement of the missing values was considered appropriate without introducing a bias in the treatment evaluation. All data were analysed according to intention-to-treat criteria (all patients randomized), except for results on somnolence, which were also analysed according to per-protocol criteria (only patients compliant with major protocol procedures).

\section{Results}

A group of 140 patients was enrolled in the study: 69 patients were randomized to levodropropizine and 71 to dihydrocodeine. Two patients in each group did not take any study medication; five other patients in the levodropropizine group and three patients in the dihydrocodeine group were withdrawn before 6 days of treatment. One patient randomized to levodropropizine (mammary carcinoma with mediastinal metastasis) and three patients randomized to dihydrocodeine (two pleural mesothelioma, one mammary carcinoma with bone metastasis and lymphangitis) were included in the deviation of inclusion criteria for underlying pathology. All these patients were excluded from the per-protocol analysis. Patients' characteristics at baseline are shown in table 1. More males than females were included, as expected, in agreement with the prevalence of lung cancer. Most of the patients within both levodropropizine and dihydrocodeine groups (between $70-80 \%$ ) were affected by primary lung cancer, including small and nonsmall cell lung cancer. The two treatment groups were homogeneous as regards to all 
Table 1. - Demographic and baseline characteristics

\begin{tabular}{|c|c|c|}
\hline Parameter & $\begin{array}{l}\text { Levodrop. } \\
\text { mean } \pm S D\end{array}$ & $\begin{array}{l}\text { Dihydrocode. } \\
\text { mean } \pm \text { SD }\end{array}$ \\
\hline Patient $n$ & 69 & 71 \\
\hline Sex M/F n (\%) & $\begin{array}{c}59 / 10 \\
(85.5 / 14.5)\end{array}$ & $\begin{array}{c}48 / 23 \\
(67.6 / 32.4)\end{array}$ \\
\hline Age yrs & $62 \pm 9$ & $64 \pm 10$ \\
\hline Weight $\mathrm{kg}$ & $67 \pm 11$ & $64 \pm 10$ \\
\hline Height $\mathrm{cm}$ & $167 \pm 7$ & $166 \pm 8$ \\
\hline Smokers n $(\%)$ & $10(14.5)$ & $10(14.1)$ \\
\hline \multicolumn{3}{|l|}{ Underlying pathology $\mathrm{n}(\%)$} \\
\hline Primary lung cancer & $54(78)$ & $53(75)$ \\
\hline $\begin{array}{l}\text { Metastatic cancer to the } \\
\text { lungs }\end{array}$ & $14(20)$ & $15(21)$ \\
\hline Other & $1(1)$ & $3(4)$ \\
\hline $\begin{array}{l}\text { Neoplastic disease duration } \\
\text { months }\end{array}$ & $15.1 \pm 29.1$ & $16.8 \pm 23.3$ \\
\hline Surgical intervention $\mathrm{n}(\%)$ & $20(29)$ & $25(35)$ \\
\hline $\begin{array}{l}\text { Cough symptom duration } \\
\text { days }\end{array}$ & $65.1 \pm 96.7$ & $40.5 \pm 41.7$ \\
\hline \multicolumn{3}{|l|}{ Cough severity score } \\
\hline Patient & $3.7 \pm 0.6$ & $3.7 \pm 0.6$ \\
\hline Investigator & $3.8 \pm 0.7$ & $3.8 \pm 0.7$ \\
\hline Night awakenings & $1.4 \pm 1.9$ & $1.1 \pm 1.5$ \\
\hline
\end{tabular}

The two groups were homogeneous as regards all demographic data and baseline features, except for sex distribution $(\mathrm{p}<0.05$, $\chi^{2}$ test). Levodrop.: levodropropizine; Dihydrocode.: dihydrocodeine; M: male; F: female.

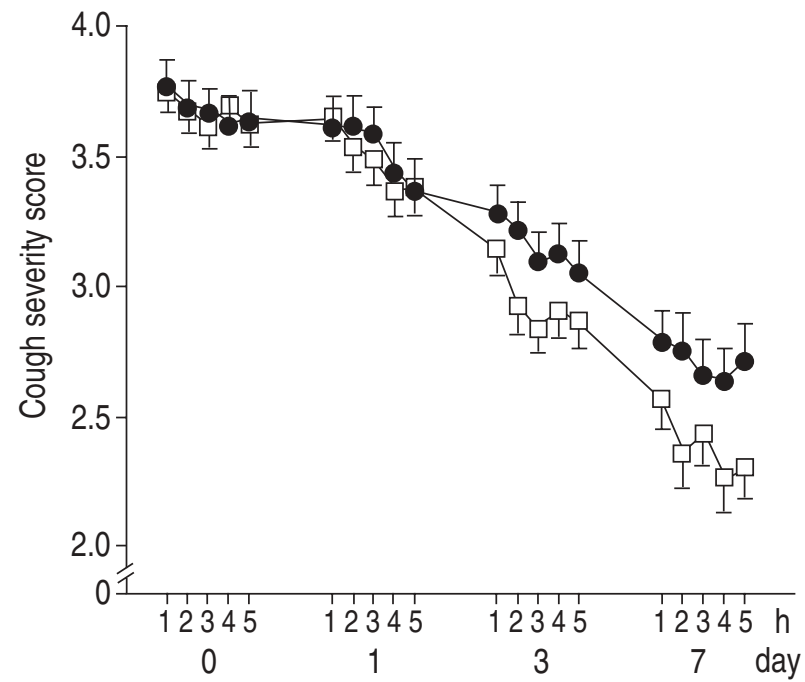

Fig. 1. - Cough severity graded by the patient. Values represent the mean \pm SEM from all available data within the levodropropizine $(n=58-67, \bullet)$ and dihydrocodeine $(n=64-68 ; \square)$ groups. Cough severity score significantly decreased $(\mathrm{p}<0.05)$ during time with both treatments, without significant difference between treatments.

demographic data and baseline features, except for sex distribution $\left(\mathrm{p}<0.05, \chi^{2}\right.$ test $)$.

Multiple antineoplastic therapy was administered during the study period in 12 patients receiving levodropropizine and in 10 patients on dihydrocodeine. Such therapy was administered in the previous 3 months before entry into the study in 15 and three patients randomized to levodropropizine and dihydrocodeine, respectively. Roentgentherapy was performed during the study period in three levodropropizine patients and in two dihydrocodeine patients; it was performed in the previous 3 months in two patients on dihydrocodeine.
The trend in cough severity score as graded by the patients is reported in figure 1 . Cough severity was significantly reduced $(\mathrm{p}<0.05)$ in both groups and the effect tended to increase with time. The time profile of cough amelioration was similar with both treatments. The trend in cough severity as judged by the investigators confirmed the patients' subjective evaluation since, also according to the investigators, both treatments produced a similar and significant decrease in cough scores $(\mathrm{p}<0.05)$ during time, with no significant difference between treatments (fig. 2).

In parallel with the positive effect on cough severity, the number of awakenings during the night in those patients with at least one night wakeup at baseline, significantly decreased $(\mathrm{p}<0.05)$ in both groups, with a similar time profile (table 2).

The overall final estimate of the efficacy further supported the antitussive activity of the two drugs. According to the patient's or investigator's judgement, cough disappeared in five $(11 \%)$ or three $(7 \%)$ patients receiving levodropropizine, and improved in $30(67 \%)$ or $33(73 \%)$. Corresponding figures for the dihydrocodeine group were five $(12 \%)$ or three $(7 \%)$ patients with cough disappearance and $31(76 \%)$ or $34(83 \%)$ with cough improvement, according to the patient's or investigator's judgement, respectively.

Somnolence was reported to be present in five out of 66 patients treated with levodropropizine and in 15 out of 69 patients treated with dihydrocodeine. The percentage of patients reporting somnolence was significantly higher

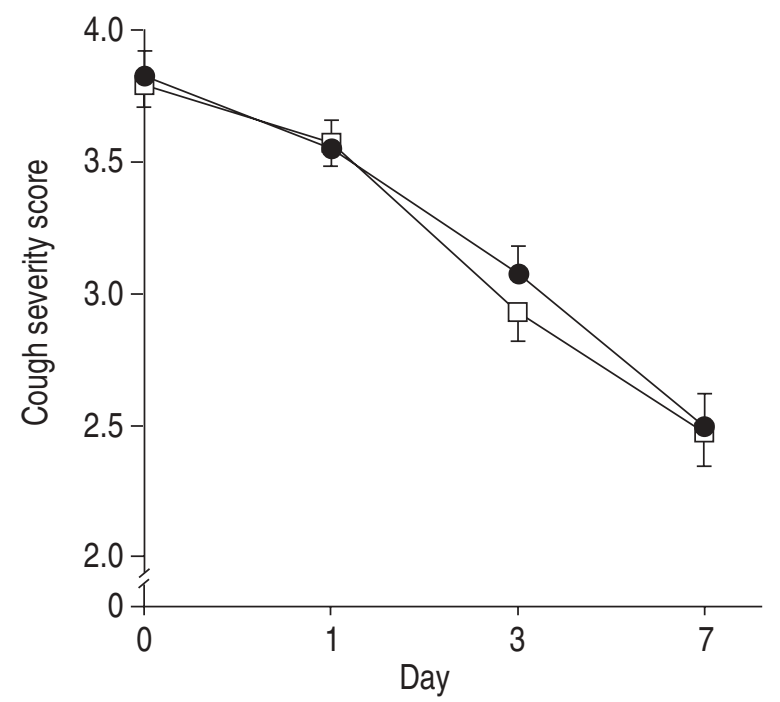

Fig. 2. - Cough severity graded by the investigator. Values represent the mean \pm SEM from all available data within the levodropropizine $(n=58-$ 69 , - ) and dihydrocodeine $(n=65-70 ; \square)$ groups. Cough severity score significantly decreased $(\mathrm{p}<0.05)$ during time with both treatments, without significant difference between treatments.

Table 2. - Night awakenings in patients with at least one night wakeup at baseline

\begin{tabular}{lccccc}
\hline \multirow{2}{*}{$\begin{array}{l}\text { Study } \\
\text { day }\end{array}$} & \multicolumn{2}{c}{ Levodropropizine } & & \multicolumn{2}{c}{ Dihydrocodeine } \\
\cline { 2 - 3 } \cline { 6 - 6 } \cline { 5 - 6 } & $\mathrm{n}$ & mean $\pm \mathrm{SD}$ & & $\mathrm{n}$ & mean \pm SD \\
\hline Baseline & 35 & $2.7 \pm 1.9$ & & 30 & $2.5 \pm 1.3$ \\
Day 1 & 34 & $2.4 \pm 2.6$ & & 29 & $1.6 \pm 1.2$ \\
Day 3 & 34 & $1.4 \pm 1.7$ & & 29 & $0.6 \pm 0.9$ \\
Day 7 & 30 & $1.2 \pm 1.7$ & & 27 & $0.6 \pm 1.1$ \\
\hline
\end{tabular}

Significance was tested by ANOVA: treatment ss; day $\mathrm{p}<0.05$; and treatment $\mathrm{x}$ day Ns. 


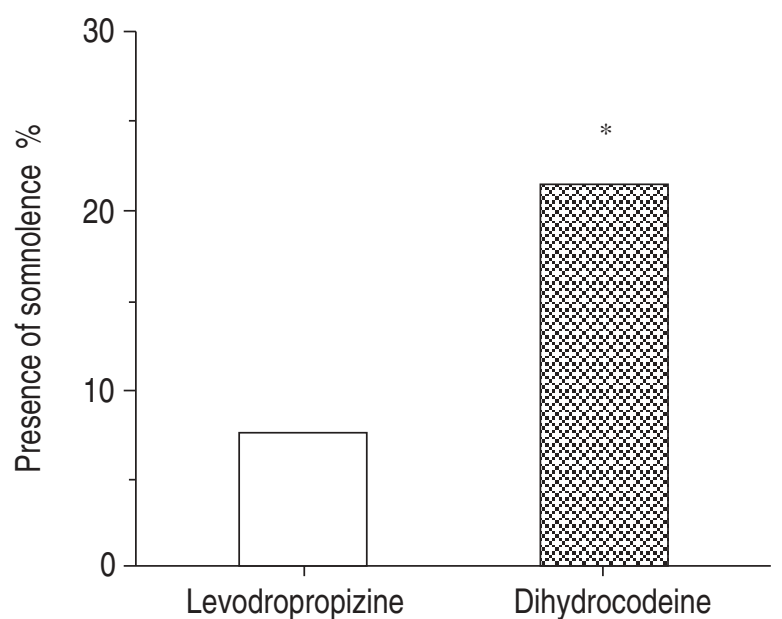

Fig. 3. - Presence of somnolence. Values represent the percentage of patients reporting somnolence within levodropropizine ( $\square$ ) and dihydrocodeine $(\mathbb{\mathbb { Q }})$ group (intention-to-treat analysis). The percentage of patients reporting somnolence during the dihydrocodeine treatment was significantly higher $(*: \mathrm{p}<0.05)$ than in the levodropropizine group.

in the dihydrocodeine group (22\%) than in the levodropropizine group $(8 \%$; $\mathrm{p}<0.05$; fig. 3$)$. No severe somnolence was recorded after treatment with either drug. Per-protocol analysis confirmed the higher prevalence of somnolence in patients receiving dihydrocodeine (15 out of $63,24 \%$ ) as compared with patients treated with levodropropizine (5 out of $60,8 \% ; \mathrm{p}<0.05$ versus dihydrocodeine). Concomitant medications known to induce somnolence were taken by three patients in the levodropropizine group and by four patients in the dihydrocodeine group.

Blood pressure, heart rate and laboratory tests did not change significantly during treatment in either group. Adverse events were reported in six patients treated with levodropropizine and in four patients treated with dihydrocodeine, including two deaths (one in each group) related to the underlying disease. In the former group, adverse events included vomiting, diarrhoea and epigastric pain, all judged to be in doubtful correlation with the study drug except for vomiting in one patient possibly related to the treatment. In the latter group, adverse events were erythema of the abdomen and epigastric pain, in doubtful correlation with the study medication, and continuous somnolence definitely related to the study drug. Constipation, a potential side-effect of codeine derivatives, was not reported with either dihydrocodeine or levodropropizine. The latter drug is, in any case, not known to induce such gastrointestinal event.

\section{Discussion}

The results of this multicentre, double-blind study demonstrated that levodropropizine reduced nonproductive cough and night awakenings due to cough in patients with primary lung cancer or metastatic cancer to the lungs. The antitussive effect increased with time and was comparable to that found during treatment with the reference drug, dihydrocodeine. However, levodropropizine induced significantly less somnolence compared to dihydrocodeine. Finally, this study confirmed that levodropropizine caused very few side-effects.
Cough, is one of the most common lung cancer-associated symptom in patients with lung cancer. Nonproductive cough is often the initial symptom [2, 14], regardless of its histological type [1,3] and affects nearly all patients. Among other factors, cough is certainly important in determining a patient's quality of life. Cough, haemoptysis, dyspnoea and chest pain are the most reliable measures for assessing both the performing status of patients and their treatment-related improvement [15].

Although cough is one of the most important defensive airway reflexes, it can be a very distressing symptom in patients with lung cancer. The presence of cough often becomes very disabling, especially in patients with a poor general condition and in those patients who continue to smoke. Since cough impairs the normal breathing pattern, it can become unbearable in patients with breathlessness, one of the other important clinical problems in lung cancer patients [4].

Neoplastic cough can be palliated by different chemotherapy regimens [16], by intraluminal radiotherapy [5] or thoracic radiotherapy in approximately half to two-thirds of patients with lung cancer. However, when different palliative approaches have been compared, including surgery, radiotherapy and simple supportive care, it appears that cough is reduced only in $34 \%$ of patients, palliation being rather poor after surgery [4]. Thus, these patients require not only specific antitumour treatments but almost invariably also a symptomatic antitussive therapy.

The frequency of cough in lung cancer and its exhausting effect have led to the search of an effective, tolerable and safe therapy. Centrally acting cough suppressants in current use are usually opiate derivatives that are presumed to act on the medullary cough centre [6]. Codeine or codeine derivatives, such as dihydrocodeine, are often used as standard antitussives against which new drugs are compared. In this study, we compared the antitussive activity of a centrally acting drug, dihydrocodeine, and that of a new peripherally acting drug, levodropropizine. Both drugs appeared to be highly and equally effective against nonproductive cough in patients with primary or metastatic lung cancer. These conclusions might be limited by the lack of a placebo control group. However, the aim of this study was the comparison between two drugs for which the efficacy has been previously demonstrated in double-blind, placebo-controlled trials $[11,12,17]$. Moreover, due to the nature of the disease, cough in lung cancer is a persistent problem that is unlikely to recover spontaneously as might be the case for acute respiratory pathologies. Indeed, patients involved in this study had a mean cough duration of more than 1 month, making it unlikely that any improvement in cough seen within a few days of treatment could be attributed to spontaneous relief.

Dihydrocodeine, besides the antitussive activity that is greater than that produced by codeine [6], also possesses an analgesic activity. This pharmacological characteristic is, indeed, linked to its effects on psychomotor as well as on cognitive functions. It has been found that these effects tend to diminish once the patient is on a stable dose, although pretreatment levels are not always achieved. A higher prevalence of somnolence in the dihydrocodeine group likely reflects this pharmacological activity. However, the lower somnolence prevalence in the levodropropizine group indirectly supports and confirms the peripheral activity of this antitussive drug [11]. In this 
regard, it should be noted that additional treatments required by patients and known to cause somnolence were equally distributed in both groups. Since side-effects were similar and trivial in both treatment groups, the results on somnolence are in favour of a better tolerability of levodropropizine in these patients. Indeed, a few patients treated with levodropropizine reported the occurrence of somnolence, which may simply reflect either a deterioration in the general conditions of the patients or the effect of additional therapy rather than an effect due to drug intake.

Mechanisms of nonproductive cough associated to lung cancer likely involve activation of non-myelinated $\mathrm{C}$ fibres through the mechanical distortion of bronchial airways and/or through the action of bradykinin. In addition, tachykinines released from $\mathrm{C}$-fibres are able to activate rapidly adapting receptors [18]. Indeed, bradykinin is commonly released by neoplastic cells [19] and neoplastic cough can be reduced by inhaled cromones through a blocking effect of bradykinin activity on C-fibres [20]. The effect of dihydrocodeine seems to be mediated by an increase in 5-HT release in the brain stem [8], whereas the antitussive activity of levodropropizine is likely due to peripheral modulation of cough receptor activity. This effect seems, at least in part, to be mediated by an interference with the neuropeptide system [11]. This hypothesis is supported by the findings that levodropropizine blocks cough induced in animals [10] and humans [21] by capsaicin, a well-known and selective stimulant for C-fibres. Moreover, levodropropizine inhibits afferent $\mathrm{C}$-fibre firing in anaesthetized cats [22] and neuropeptide release from pulmonary C-fibres in isolated guinea-pig lungs [23]. Thus, it is possible that this mechanism might account also for the effectiveness of levodropropizine against cough in pat-ients with lung cancer.

In conclusion, this study demonstrated that levodropropizine and dihydrocodeine were equally effective in relieving nonproductive cough in most patients with primary lung cancer or metastatic cancer to the lungs. Side-effects were also comparable, with the exception of somnolence that was reported less frequently during levodropropizine than during dihydrocodeine treatment.

Participating investigators: G. Luporini, M. Clerici, Divisione di Oncologia Medica, Ospedale S. Carlo Borromeo, Milano; E. Marchi, Centro per lo Studio delle Broncopneumopatie Senili, Casatenovo (LC); R. Labianca, G. Beretta, Sezione di Oncologia Medica, Ospedale E. Bassini, Cinisello Balsamo (MI); S. Barni, Day Hospital - Oncologia Medica, Ospedale S. Gerardo, Monza (MI); E. Soresi, Divisione di Fisiopatologia Respiratoria, Ospedale Niguarda Ca' Granda, Milano; R. Rimoldi, Divisione di Pneumologia, Ospedale di Circolo, Varese; F. Berni, Divisione di Pneumologia, Ospedale S.ta Corona, Garbagnate (MI); E. Farina, Divisione di Pneumologia, Ospedale S. Salvatore, L'Aquila.

\section{References}

1. Sridhar KS, Bounassi MJ, Raub W Jr, et al. Clinical features of adenosquamous lung carcinoma in 127 patients. Am Rev Respir Dis 1990; 142: 19-23.

2. Anonymous. A Medical Research Council (MRC) randomised trial of palliative radiotherapy with two fractions or a single fraction in patients with inoperable non-smallcell lung cancer (NSCLC) and poor performance status. Medical Research Council Lung Cancer Working Party. Br J Cancer 1992; 65: 934-941.

3. Hopwood P, Stephens RJ. Symptoms at presentation for treatment in patients with lung cancer: implications for the evaluation of palliative treatment. The Medical Rese- arch Council (MRC) Lung Cancer Working Party. Br J Cancer 1995; 71: 633-636.

4. Muers MF, Round CE. Palliation of symptoms in nonsmall cell lung cancer: a study by the Yorkshire Regional Cancer Organisation Thoracic Group. Thorax 1993; 48: 339-343.

5. Gollins SW, Burt PA, Barber PV, et al. High dose rate intraluminal radiotherapy for carcinoma of the bronchus: outcome of treatment of 406 patients. Radiother Oncol 1994; 33: 31-40.

6. Braga PC, Allegra L. Centrally acting opioid drugs. In: Braga PC, Allegra L, eds. Cough. New York, Raven Press, 1989; pp. 109-140.

7. O'Neil WM. The cognitive and psychomotor effects of opioid drugs in cancer pain management. Cancer Surv 1994; 21: 67-84.

8. Kamei J, Mori T, Igarashi $\mathrm{H}$, et al. Serotonin release in nucleus of the solitary tract and its modulation by antitussive drugs. Res Commun Chem Pathol Pharmacol 1992; 76: 371-374.

9. Munt PL, Clavenna G, Algate DR, et al. Antitussive effects of levodropropizine in the dog. Arzneim-Forsch Drug Res 1994; 44: 153-155.

10. Malandrino S, Melillo G, Bestetti A, et al. Antitussive properties of levodropropizine. Arzneim-Forsch Drug Res 1988; 38: 1144-1150.

11. Daffonchio L, Clavenna G, Fedele G, et al. Levodropropizine. Drugs Today 1995; 31: 299-305.

12. Guffanti EE. Drugs with direct peripheral action. In: Braga PC, Allegra L, eds. Cough. New York, Raven Press, 1989; pp. 198-214.

13. Banderali G, Riva E, Fiocchi A, et al. Efficacy and tolerability of levodropropizine and dropropizine in children with non-productive cough. J Int Med Res 1995; 23: 175-183.

14. Anonymous. Inoperable non-small cell lung cancer (NSCLC): a Medical Research Council randomised trial of palliative radiotherapy with two fractions of ten fractions. Report to the Medical Research Council by its Lung Cancer Working Party. Br J Cancer 1991; 63: 265-270.

15. Bergman B, Aaronson NK, Ahmedzai S, et al. The EORTC QLQ-LC13: a modular supplement to the EORTC Core Quality of Life Questionnaire (QLQ-C30) for use in lung cancer clinical trials. EORTC Study Group on Quality of Life. Eur J Cancer 1994; 30A: 635-642.

16. Bleehen NM, Girling DJ, Machin D, et al. A randomised trial of three or six courses of etoposide cyclophosphamide methotrexate and vincristine or six courses of etoposide and ifosfamide in small cell lung cancer (SCLC). II: Quality of life. Medical Research Council Lung Cancer Working Party. Br J Cancer 1993; 68: 1157-1166.

17. Matthys H, Erhardt J, Ruhle KH. Objektivierung der wirkung von antitussiva mittels tussometrie an patienten mit chronischem husten. Schweiz, Med Wochrnschr 1985; 115(9): 307-311.

18. Kaufman MP, Coleridge KIM, Coleridge JC, et al. Bradykinin stimulates afferent vagal C-fibers in intrapulmonary airways of dogs. J Appl Physiol 1980; 48: 511-517.

19. Bunn PAJ, Chan D, Dienhart DG, et al. Neuropeptide signal transduction in lung cancer clinical implications of bradykinin sensitivity and overall heterogeneity. Cancer Res 1992; 52: 24-31.

20. Moroni M, Porta C, Gualtieri G, et al. Inhaled sodium cromoglycate to treat cough in advanced lung cancer patients. Br J Cancer 1996; 74: 309-311.

21. Mistretta A, Privitera S, Pulvirenti G, et al. Protective effect of levodropropizine against capsaicin-induced cough in allergic patients: a double-blind, placebo-controlled study. Adv Ther 1992; 9: 98-106.

22. Shams H, Daffonchio L, Scheid P. Effects of levodropropizine on vagal afferent C-fibres in the cat. Br J Pharmacol 1996; 117: 853-858.

23. Daffonchio L, Geppetti P, Javdan P, et al. Mechanism of neuropeptide system inhibition in the lung: a study with levodropropizine. Pharmacol Res 1995; 31 (Suppl): 309. 